\title{
Disgusting foods, pure water and beautiful truth for balanced muscle control
}

\begin{abstract}
The author proposes that conscious awareness of disgust or disgust exercises can decrease tremors, and muscle rigidity, as well as support other functions of the basal ganglia, insula, hypothalamus, and the cerebellum. Pattern recognition skills needed to experience and identify disgust in the faces of others is compromised in people with brain dysfunctions like depression, bipolar disorder, associated with sensory dysfunctions and mood, Huntington's disease (a genetic movement disorder), focal dystonia (unusually tight twisted muscles), Parkinson's disease (tremor and movement disorder), Wilson's disease (genetic copper processing disorder), and Velo-cardio-facial syndrome (a genetic disorder associated with interstitial deletions of chromosome 22q11.2). Disgust exercises or consciously paying attention to what is disgusting and what is pleasant or beautiful may trigger changes in the basal ganglia, where disgust is perceived and lead to improvements in balance, walking, tremors, obsessive compulsive disorder symptoms, depression, and overall brain function. The different functions of a particular brain structure can be considered doorways into healing. The basal ganglia and the neurochemical dopamine have responsibilities for disgust recognition, suppressing tremors as well as other unwanted muscle movements by balancing motor input from other areas of the brain, and preventing muscle rigidity.
\end{abstract}

Keywords: disgust, brain health, basal ganglia, dopamine, parkinson's, tremors, huntington's ataxia, balance
Volume 2 Issue 3 - 2015

\author{
Kimberly B \\ Complementary Medicine, Akamai University, USA
}

Correspondence: Burnham K, Akamai University, 3608 South Fancher Road, Spokane,WA, USA , Tel (860) 221-8510, Email Nervewhisperer@gmail.com

Received: October 24, 2015 | Published: : December 31, 2015
Abbreviations: $\mathrm{BD}$, bipolar disorder; COMT, catecholo-methyl transferase; HD, huntington's disease; OC, obsessive compulsive; PD,parkinson's disease; VCFS, velo-cardio-facial syndrome

\section{Disgusting foods and beautiful truth for balanced muscle control}

Our environment is created by our choices in the areas of diet, lifestyle, exercise, and more. Knowing that these choices make a difference in our quality of life, even in genetically based conditions, helps people consider their choices, stick to plans, and recover quality of life. All of this can be supported and facilitated by a great health coach. Imagine stringy, smelly, fermented soy beans-Japanese natto. Third culture kids, like me have expanded our definition of disgusting foods by traveling widely, sampling mushy grey gruel in food cars on Chinese trains or sharp pungent onion salad, the only "greens" available on the Trans-Siberian railway or even spoiled key lime pie in Toronto, Canada sitting out in the summer heat, curdling my taste buds.

In Japan, no one ever says, "yeah, natto it is okay. I am fine with or without it." You either hate it or, and I can't understand this part, love it. Seriously, there are people whose favorite food is brown, slimy natto. It's super healthy for your heart but only if you can keep it down.

Taking about another Japanese food, "Sushi" explains an avid fisherman, "has an English translation-bait." And yet there are millions of people worldwide who love fresh raw tuna. Me, I can't stand the feel of it sliding down my throat, even after four years of living in Japan.

What disgusting thing have you eaten lately? Come on, we have all eaten disgusting bits of food or even done something disgusting. But did you know, disgust, a beneficial emotion, sometimes saves us from food poisoning and helps us sort out what is true in our lives?
And if this is an average day in the United States, thirteen people will die from food poisoning.

Here we will look at conscious awareness of disgust and how it can not only save us from food poisoning but also improve brain health.

Global travelers have also experienced some of the loveliest foods in the world. It has been nearly fifty years. I still remember the sweet creamy texture of a tree-ripened mango in Bogota, Colombia. Sitting atop a slippery slide in our walled backyard, juice dripped down my chin, staining my blue and white checked shirt. With my teeth, I scraped off every last bit of yellow-orange flesh before following the big white pit down the slide and running inside for another mango. ${ }^{1}$

We value certain foods much higher than others, with a part of the brain called the basal ganglia. Part of the subcortical brain, the basal ganglia and the hypothalamus form a link between goal directed behavior and habit forming rewards. Damage to the basal ganglia disrupts our ability to achieve goals, earn specific rewards, or avoid disgusting experiences. With brain damage, the carrot and the stick are more difficult to achieve or said another way, natto and mangos seem more similar.

Goal directed behavior also involves the basal ganglia, which is involved in cognitively controlled timing systems that requires attention. "Time is a fundamental dimension of life. It is crucial for decisions about quantity, speed of movement, and rate of return, as well as for motor control in walking, speech, appreciating music, and participating in sports." Buhusi ${ }^{2}$ added, "It is now proposed that the brain represents time in a distributed manner and tells the time by detecting the coincidental activation of different neural populations." Perhaps an inability to tell funny jokes is a sign of basal ganglia dysfunction or practicing joke telling can improve this part of the brain. The basal ganglia has pathways that also carries the balance skills and ways to control the fine movements needs to be a ballet dancer or concert violinist. It controls our sensations, muscle movement, ${ }^{3}$ and internal states-how we feel inside and how flexible we are in our choices. 
Goal driven, we can seek out new foods, new experiences, or habitually order our same old favorite foods in restaurants. Maybe we are addicted to certain foods. Researchers call this a maladaptive type of habit learning or maybe we have an appetitive Pavlovian conditioning to certain foods we grew up with. Driven by the limbic system part of the brain we respond to a particular stimulus, like the smell of apple pie in the same way over and over. ${ }^{4}$ This might not seem like such a big deal but "the adaptive significance of disgust has been related to a specific form of threat response associated with an internal defense system, as opposed to an external defense system related to fear," according to, Adolphs ${ }^{5}$ in "Neural Systems for Recognizing Emotions From Facial Expressions." This means our emotions and ability to read the emotional states of other people keeps us safe-safe from internal things like food poisoning and from external things like bears. In other words our ability to feel and recognize disgust keeps us safe from the inside out.

Think of your brain influenced food choices, as bright yellow buses on a highway. You can achieve your goals and get where you are going, on whichever one you want. You get to choose. By choosing wisely, you improve the journey, the road, and become more functional. Choose unwisely and you get food poisoning or ostracized from the community. In a similar way to our choices, walking, and taking fish oils improves brain function. Consciously noticing what disgusts or excites can lay down shiny new neural pathways and create memories that last a lifetime. Pattern recognition skills needed to experience and identify disgust in the faces of others is compromised in people with brain dysfunctions like depression, Huntington's disease (a genetic movement disorder), focal dystonia (unusually tight twisted muscles), Parkinson's disease (tremor and movement disorder), Wilson's disease (genetic copper processing disorder), Velo-cardio-facial syndrome VCFS (a genetic disorder associated with interstitial deletions of chromosome 22q11.2), and bipolar disorder (BD), associated with sensory dysfunctions and mood. And it is not just visual recognition. In one study, participants listened to emotional sentences expressing one of four emotions (anger, fear, disgust, happiness) or neutral sentences. People with problems of the basal ganglia "misclassified neutral sentences as disgust sentences more often than healthy controls," said Paulmann et al., "Emotional Speech Perception Unfolding in Time: The Role of the Basal Ganglia." In a Parkinson's disease and dopaminergic pathways study, researchers noted, "results showed that the early Parkinson's disease patients performed more poorly in the $\mathrm{ON}$ medications condition than in the OFF one, for overall emotion recognition, as well as for the recognition of anger, disgust, and fear. Additionally, for anger, the early Parkinson's ON patients performed more poorly than controls. For overall emotion recognition, both advanced Parkinson's patients and early Parkinson's ON patients performed more poorly than controls. These results confirm the involvement of the dopaminergic pathways and basal ganglia in emotional prosody recognition, and suggest a possibly deleterious effect of dopatherapy on affective abilities in the early stages of Parkinson's," according to Peron et al., in "Effect of Dopamine Therapy on Nonverbal Affect Burst Recognition in Parkinson's Disease." Dopamine is needed in the basal ganglia in order to help us read other people's emotional states as well as to suppress tremors. In people with Parkinson's disease a lack of dopamine results in tremors and other unwanted movements. Problems with dopamine and its relationship to disgust and fear is described in this 2015 article by Azuma et al., "We selected fear and disgust out of the basic human emotional expressions because of their significance in the development of socialization skills," according to the Journal of Neurodevelopmental Disorders article, "An fMRI Study of Facial Emotion Processing in Children and Adolescents with 22q11.2 Deletion Syndrome." Researchers continued, "One possible explanation for differences in modulation of social brain regions by different types and intensity of facial expression may be variation in dopamine metabolism-for example, associated with variation in catechol-O-methyl transferase (COMT), a methylation enzyme that metabolizes catecholamines (including dopamine). "In other words, dopamine levels in social brain regions could be abnormal in people with 22q11DS. Other symptoms of the genetic condition include, heart defects, speech and language delay, developmental delay (including difficulties with social skills), palate problems, poor immunity to infections, emotional, behavioral and psychiatric issues, learning differences, feeding difficulties, and autistic tendencies. What if these symptoms could change depending on what we pay attention to and how we view the people and world around us? Dopamine is the neurochemical of the reward system. It is also the neurochemical associated with the placebo effect. If we think we are going to heal, get a reward, or be served something disgusting, dopamine levels change. In a 2015 article in Nature Reviews Neuroscience, Wager et al., said, "Placebo effects are beneficial effects that are attributable to the brain-mind responses to the context in which a treatment is delivered rather than to the specific actions of the drug. They are mediated by diverse processes--including learning, expectations and social cognition--and can influence various clinical and physiological outcomes related to health. Emerging neuroscience evidence implicates multiple brain systems and neurochemical mediators, including opioids and dopamine."

Our ability to recognize rewards, disgust, and other emotions is linked to how we feel. There are sensory exercises that can impact how we feel and experience the world. Vuillier et al. ${ }^{10}$ recently said, "Emotional processing has been reported to effect sensory gating as measured by the event-related potential known as P50. Because both P50 and emotional processing are dysfunctional in bipolar disorder (BD), we sought to investigate the impact that concurrent emotional processing has on sensory gating in this psychiatric population." Researchers added, "The bipolar disorder group showed significant associations between sensory gating to disgust and measures of social functioning. Importantly, bipolar disorder showed impaired filtering of auditory information when paired with an emotionally salient image. Thus, it appears that patients with the greatest impairment in sensory gating also have the most difficulty engaging in social situations."

Several diseases with psychological symptoms, facial emotion recognition, and movement disorder symptoms are correlated with dopamine. Parkinson's disease (too little) and schizophrenia (too much). can be considered to be on opposite ends of the same spectrum. Yang et al. ${ }^{11}$ noted, "Although many studies have examined executive functions and facial emotion recognition in people with schizophrenia, few of them focused on the correlation between them. Furthermore, their relationship in the siblings of patients also remains unclear. Our study demonstrated that facial emotion recognition impairment correlated with executive function impairment in people with schizophrenia and their unaffected siblings but not in healthy controls."

Awareness and consciously considering what is disgusting is one way to help heal the brain. Think of disgust exercises as weight training for several key functions of the brain.

In addition to your brain's culinary ability, researchers Harris et al., ${ }^{12}$ Annals of Neurology noted another function of the basal ganglia. "The difference between believing and disbelieving a proposition is one of the most potent regulators of human behavior and emotion." Whether we accept something as true or reject it as a false string of words, depends on the health of our brain. Continuing he says, "Truth may be beauty, and beauty truth, in more than a metaphorical 
sense, and false propositions may actually disgust us.” This idea also pertains to the placebo effect, where what a person believes about the treatment, pill, or health care professional matters. Or as is said in quantum physics, the observer matters. How we look at the people and the world around us changes everything.

In a 2014 article in the International Journal of Psychology researchers noted, "Increased disgust sensitivity has also been reported to be associated with obsessive compulsive (OC) symptoms. No research, however, has investigated the mediating roles of thought-action fusion and disgust sensitivity between religiosity and obsessive compulsive symptoms. This study was composed of 244 undergraduate students who completed measures of obsessive compulsive symptoms, thought-action fusion, disgust sensitivity, religiosity and negative effect. Analyses revealed that the relationship between religiosity and obsessive compulsive symptoms was mediated by thought-action fusion and disgust sensitivity. More importantly, the mediating role of thought-action fusion was not different across obsessive compulsive symptom subtypes, whereas the mediating role of disgust sensitivity showed different patterns across obsessive compulsive symptom subtypes. These findings indicate that the tendency for highly religious Muslims to experience greater obsessive compulsive symptoms is related to their heightened beliefs about disgust sensitivity and the importance of thoughts," according to Inozu et al. ${ }^{13}$ in "The Mediating Role of Disgust Sensitivity and Thought-Action Fusion Between Religiosity and Obsessive Compulsive Symptoms."

Research seems to indicate that drinking clean water and staying hydrated can also help with brain function. Meier et al., ${ }^{14}$ in "Rivalry of homeostatic and sensory-evoked emotions: Dehydration attenuates olfactory disgust and its neural correlates," said "Neural correlates have been described for emotions evoked by states of homeostatic imbalance (e.g. thirst, hunger, and breathlessness) and for emotions induced by external sensory stimulation (such as fear and disgust). " The study showed that the sensory function of the brain was impaired in cases of dehydration. Thirst lead to a lack of survival responses or the ability to keep one's self safe. "Twenty highly dehydrated male subjects rated a disgusting odor as significantly less repulsive when they were thirsty. On the neurobiological level, we found that this reduction in subjective disgust during thirst was accompanied by a significantly reduced neural activity in the insular cortex, a brain area known to be considerably involved in processing of disgust. Furthermore, during the experience of disgust in the satiated condition, we observed a significant functional connectivity between brain areas responding to the disgusting odor, which was absent during the stimulation in the thirsty condition. "

It is interesting to note that in people with Huntington's disease, which affects the basal ganglia and hypothalamus there is an alteration in thirst and water consumption. Wood et al., ${ }^{15}$ in "Increased Thirst and Drinking in Huntington's Disease," said, "While Huntington's disease (HD) is a condition that primarily involves the basal ganglia, there is evidence to suggest that the hypothalamus is also affected. Because the osmoreceptors regulating thirst are situated in the circumventricular region of the hypothalamus, we were interested in whether altered thirst is a part of the Huntington's disease phenotype. We gave a xerostomia (dry mouth) questionnaire to Huntington's disease patients and control subjects, and also measured their urine osmolality and serum vasopressin. The mean total xerostomia score was significantly higher in Huntington's disease patients than in controls, indicating greater thirst in Huntington's disease patients. Urine osmolality was unaffected in Huntington's disease patients up to clinical stage III, and none of the patients had diabetes. However, serum vasopressin was increased, suggesting a dysregulation in the control of hypothalamic vasopressin release. A dry mouth can affect taste, mastication and swallowing, all of which may contribute to the significant weight loss seen in both Huntington's disease patients."

Sometimes we do disgusting things on purpose, like the time I drank some cactus juice that went down like thick gritty pulp but then ... I take in the color, the drums, the cactus, the smoky air, and touch the world around me. I have enhanced my gut sense, intuition, and brain clarity. I reach for the sunlight and the star light. I am perfectly placed, perfectly aligned to walk into the unknown with clarity and hope. My new sense of the world stay with me long after the disgust in my belly has gone. It is interesting to note the origin of the word disgust and its relationship with digestion and gustatory function.

Even though as a child I loved mangos, for a few years in the 1980's I couldn't eat anything that tasted like mango because in Malaysia, I got sick drinking mango flavored water. Now, my rational mind knew it was probably some contamination in the water not the mango powder but my body carried a powerful memory of the last thing I drank before starting to throw up. I literally couldn't even smell a mango without that nauseas feeling coming back. It was compounded by the fact that I was on vacation on Tioman Island with a bad sunburn, throwing up sick, no appetite for spicy, the-only-food-available, sleeping in a little hut on the beach with sand and who knows what else in the sheets, and an Asian style squatty potty a ways down the beach. But, I still remember the beautiful sunsets and my partner looking at me and saying, with a laugh, "Just think, people at home are envying us." The way complementary medicine practitioners and health coaches can use this information is two-fold. One is to know that although Huntington's is a genetically based disorder, studies with identical twins (hence identical genetics) shows that there are environmental factors that influence quality of life and the progression of the disease. One identical twin may have a different symptom picture and progression from the other twin. Our environment is created by our choices in the areas of diet, lifestyle, exercise and more. Knowing that these choices make a difference in our quality of life, even in genetically based conditions, helps people consider their choices, stick to plans, and all of this can be supported and facilitated by a great health coach.

The second way is to create a questionnaire or individual coaching program for the client with the coach that weird as it sounds encouraging them to think about disgusting things for a few minutes a day. This is a sample plan.

\section{Exercise: disgust: using the mind to heal the brain}

Take about 10 to 30 minutes to do this exercise. First, notice how you feel-your mood and connection to your sensory environment. Walk around a little, and notice how you are walking, or how your balance is, etc. Check in with yourself. Then answer these questions. Then recheck how you are feeling and notice what has changed. What is changed by this exercise and the attention your pay?

\section{1 . What is the most disgusting food you have ever eaten?}

2. What foods do other people like that you find disgusting?

3. What was the last disgusting smell you experienced?

4. What has a disgusting feeling (touch or texture on your skin or in your mouth)?

5. Whose face did you see with a look of disgust most recently?

6. If you imagine someone offering you something that looks disgusting, how will you respond to them? 


\section{Acknowledgments}

None.

\section{Conflicts of interest}

None.

\section{Funding}

None.

\section{References}

1. Woolley JD, EV Strobl Virginia ES, Tal Shany-Ur, et al. Impaired Recognition and Regulation of Disgust Is Associated with Distinct but Partially Overlapping Patterns of Decreased Gray Matter Volume in the Ventroanterior Insula. Biol Psychiatry. 2015;78(7):505-514.

2. Buhusi CV, WH Meck. What makes us tick? Functional and neural mechanisms of interval timing. Nat Rev Neurosci. 2005;6(10):755-765.

3. Douglas KM, RJ Porter. Recognition of disgusted facial expressions in severe depression. Br J Psychiatry. 2010;197(2):156-157.

4. Smeets PA, Charbonnier L, van Meer F, et al. "Food-induced brain responses and eating behaviour. Proc Nutr Soc. 2012;71(4): 511-520.

5. Adolphs R. Neural systems for recognizing emotion. Curr Opin Neurobiol. 2002;12(2):169-177.

6. Paulmann SDV, Ott DV, Kotz SA. Emotional speech perception unfolding in time: the role of the basal ganglia. PLoS One. 2011;6(3):e17694.
7. Péron J, Grandjean D, Drapier S, et al. Effect of dopamine therapy on nonverbal affect burst recognition in Parkinson's disease. PLoS One. 2014;9(3):e90092

8. Azuma RQ, Deeley Q, Linda EC, et al. An fMRI study of facial emotion processing in children and adolescents with 22q11.2 deletion syndrome. J Neurodev Disord. 2015;7(1):1-16.

9. Wager TD, Atlas LY. The neuroscience of placebo effects: connecting context, learning and health. Nat Rev Neurosci. 2015;16(7):403-418.

10. Vuillier L, Hermens DF, Kate C, et al. Emotional processing, p50 sensory gating, and social functioning in bipolar disorder. Clin EEG Neurosci. 2015;46(2):81-87.

11. Yang C, Zhang T, Li Z, et al. The relationship between facial emotion recognition and executive functions in first-episode patients with schizophrenia and their siblings. BMC Psychiatry. 2015; 15(1):241.

12. Harris S, Sheth SA, Cohen MS. Functional neuroimaging of belief, disbelief, and uncertainty. Ann Neurol. 2008;63(2):141-147.

13. Inozu M, Ulukut FO, Ergun G, et al. The mediating role of disgust sensitivity and thought-action fusion between religiosity and obsessive compulsive symptoms. Int J Psychol. 2014;49(5):334-341.

14. Meier L, Friedrich H2, Federspiel A3, et al. Rivalry of homeostatic and sensory-evoked emotions: Dehydration attenuates olfactory disgust and its neural correlates. Neuroimage. 2015;114:120-127.

15. Wood NI, Goodman AO, van der Burg JM, et al. Increased thirst and drinking in Huntington's disease and the R6/2 mouse. Brain Res Bull. 2008;76(1-2):70-79. 